\title{
RESEARCH
}

Open Access

\section{MRI predictors of amyloid pathology: results from the EMIF-AD Multimodal Biomarker Discovery study}

Mara ten Kate ${ }^{1^{*+}} \mathbb{D}$, Alberto Redolfi ${ }^{2 \dagger}$, Enrico Peira ${ }^{2}$, Isabelle Bos ${ }^{3}$, Stephanie J. Vos ${ }^{3}$, Rik Vandenberghe ${ }^{4,5}$, Silvy Gabel ${ }^{4,5}$, Jolien Schaeverbeke ${ }^{4,5}$, Philip Scheltens ${ }^{1}$, Olivier Blin ${ }^{6}$, Jill C. Richardson ${ }^{7}$, Regis Bordet ${ }^{8}$, Anders Wallin ${ }^{9}$, Carl Eckerstrom ${ }^{9}$, José Luis Molinuevo ${ }^{10}$, Sebastiaan Engelborghs ${ }^{11,12}$, Christine Van Broeckhoven ${ }^{13,14}$, Pablo Martinez-Lage ${ }^{15}$, Julius Popp ${ }^{16,17}$, Magdalini Tsolaki ${ }^{18}$, Frans R. J. Verhey ${ }^{3}$, Alison L. Baird ${ }^{19}$, Cristina Legido-Quigley ${ }^{20}$, Lars Bertram ${ }^{21,22,23}$, Valerija Dobricic ${ }^{21}$, Henrik Zetterberg ${ }^{24,25,26,27}$, Simon Lovestone ${ }^{19}$, Johannes Streffer ${ }^{11,28}$, Silvia Bianchetti ${ }^{2}$, Gerald P. Novak ${ }^{29}$, Jerome Revillard ${ }^{30}$, Mark F. Gordon ${ }^{31,32}$, Zhiyong Xie ${ }^{33}$, Viktor Wottschel ${ }^{34}$, Giovanni Frisoni ${ }^{2,35}$, Pieter Jelle Visser ${ }^{1,3}$ and Frederik Barkhof ${ }^{34,36}$

\begin{abstract}
Background: With the shift of research focus towards the pre-dementia stage of Alzheimer's disease (AD), there is an urgent need for reliable, non-invasive biomarkers to predict amyloid pathology. The aim of this study was to assess whether easily obtainable measures from structural MRI, combined with demographic data, cognitive data and apolipoprotein E (APOE) \&4 genotype, can be used to predict amyloid pathology using machine-learning classification.

Methods: We examined 810 subjects with structural MRI data and amyloid markers from the European Medical Information Framework for Alzheimer's Disease Multimodal Biomarker Discovery study, including subjects with normal cognition (CN, $n=337$, age $66.5 \pm 7.2,50 \%$ female, 27\% amyloid positive), mild cognitive impairment (MCl, $n=375$, age 69 . $1 \pm 7.5,53 \%$ female, $63 \%$ amyloid positive) and AD dementia ( $n=98$, age $67.0 \pm 7.7,48 \%$ female, $97 \%$ amyloid positive). Structural MRI scans were visually assessed and Freesurfer was used to obtain subcortical volumes, cortical thickness and surface area measures. We first assessed univariate associations between MRI measures and amyloid pathology using mixed models. Next, we developed and tested an automated classifier using demographic, cognitive, MRI and APOE $\varepsilon 4$ information to predict amyloid pathology. A support vector machine (SVM) with nested 10-fold cross-validation was applied to identify a set of markers best discriminating between amyloid positive and amyloid negative subjects.
\end{abstract}

Results: In univariate associations, amyloid pathology was associated with lower subcortical volumes and thinner cortex in AD-signature regions in CN and $\mathrm{MCl}$. The multi-variable SVM classifier provided an area under the curve (AUC) of $0.81 \pm 0$. 07 in $\mathrm{MCl}$ and an AUC of $0.74 \pm 0.08$ in CN. In CN, selected features for the classifier included APOE $\varepsilon 4$, age, memory scores and several MRI measures such as hippocampus, amygdala and accumbens volumes and cortical thickness in temporal and parahippocampal regions. In MCl, the classifier including demographic and APOE $\varepsilon 4$ information did not improve after additionally adding imaging measures.

(Continued on next page)

\footnotetext{
* Correspondence: m.tenkate1@vumc.nl

${ }^{\dagger}$ Mara ten Kate and Alberto Redolfi contributed equally to this work.

'Alzheimer Center \& Department of Neurology, VU University Medical Center,

PO Box 7057, 1007 MB Amsterdam, the Netherlands

Full list of author information is available at the end of the article
}

(c) The Author(s). 2018 Open Access This article is distributed under the terms of the Creative Commons Attribution 4.0 International License (http://creativecommons.org/licenses/by/4.0/), which permits unrestricted use, distribution, and

reproduction in any medium, provided you give appropriate credit to the original author(s) and the source, provide a link to the Creative Commons license, and indicate if changes were made. The Creative Commons Public Domain Dedication waiver (http://creativecommons.org/publicdomain/zero/1.0/) applies to the data made available in this article, unless otherwise stated. 
(Continued from previous page)

Conclusions: Amyloid pathology is associated with changes in structural MRI measures in CN and $\mathrm{MCl}$. An automated classifier based on clinical, imaging and APOE $\varepsilon 4$ data can identify the presence of amyloid pathology with a moderate level of accuracy. These results could be used in clinical trials to pre-screen subjects for anti-amyloid therapies.

Keywords: Alzheimer's disease, Mild cognitive impairment, Biomarkers, Magnetic resonance imaging, Amyloid, Machine learning, Support vector machine, European Medical Information Framework for Alzheimer's Disease

\section{Background}

Alzheimer's disease (AD) is characterized pathologically by beta-amyloid $(A \beta)$ plaques and neurofibrillary tangles of misfolded tau protein [1]. As amyloid pathology may arise up to two decades before the onset of dementia, research focus has shifted towards the pre-dementia stage, which provides an opportunity for secondary prevention [2-4]. The design of clinical trials targeting the amyloid pathway in this early stage would be facilitated by the ability to recruit subjects with amyloid pathology. Amyloid pathology can be assessed in cerebrospinal fluid (CSF), obtainable by lumbar puncture, or on positron emission tomography (PET) scans. However, obtaining CSF is relatively invasive and PET scans are costly, invasive by exposing subjects to radiation and are not universally available. As the estimated prevalence of amyloid pathology between the ages of 60 and 80 ranges from 10 to $33 \%$ for cognitively normal $(\mathrm{CN})$ subjects and from 37 to $60 \%$ for subjects with mild cognitive impairment (MCI) [5], assessing amyloid pathology with CSF or PET for screening purposes is likely inefficient. Finding minimally invasive biomarkers predicting amyloid pathology could reduce the number of invasive, costly and time-consuming measures in clinical trials.

Brain atrophy markers derived from structural magnetic resonance imaging (MRI) could serve as a potential biomarker for amyloid pathology [6-12]. In this study, we evaluate the use of easily obtainable MRI measures for the prediction of amyloid pathology. We included both visual rating scores, which can be easily performed in clinical settings, and quantitative measures of subcortical volumes, cortical thickness and surface area, which can be derived from freely available software and may be more sensitive than visual ratings. We first assessed univariate associations between MRI measures and amyloid pathology. Next, we used support vector machine (SVM) analysis to develop a multi-variable classifier for predicting brain amyloid pathology at a single subject level. Besides imaging measures, we also included other non-invasive measures relevant to $\mathrm{AD}$ in the classifier, including demographic information, cognitive testing and apolipoprotein $\mathrm{E}(A P O E) \varepsilon 4$ genotype.

\section{Methods}

\section{Participants}

We included participants from the European Medical Information Framework for Alzheimer's Disease Multimodal
Biomarker Discovery (EMIF-AD MBD) study. The aim of this study was to discover novel diagnostic and prognostic markers for pre-dementia $\mathrm{AD}$, by making use of existing data and samples [13]. The EMIF-AD MBD study pooled data of $494 \mathrm{CN}, 526 \mathrm{MCI}$ and $201 \mathrm{AD}$-dementia participants from three multicentre and eight single-centre studies. Inclusion criteria were: presence of normal cognition, MCI or a clinical diagnosis of AD-type dementia; availability of data on amyloid pathology, measured in CSF or on PET; age above 50 years; availability of MRI scans, plasma, DNA or CSF samples (at least two of the modalities); and absence of major neurological, psychiatric or somatic disorders that could cause cognitive impairment.

From the 1221 subjects included in the EMIF-AD MBD study, MRI scans of 873 subjects were contributed by the different studies (Fig. 1). Based on visual assessment, 863 MRI scans were of sufficient quality for visual rating, consisting of $365 \mathrm{CN}, 398 \mathrm{MCI}$ and 100 AD-dementia participants. Data were obtained from the following cohorts: DESCRIPA [14], EDAR [15], PharmaCog [16] and single-centre studies at VU University Medical Centre [17], San Sebastian GAP [18], University of Antwerp [19], Leuven [20], University of Lausanne [21], University of Gothenburg [22] and Barcelona IDIBAPS [23]. Each study was approved by the local medical ethics committee. Subjects had provided written informed consent at the time of inclusion in the MBD study for sharing of data, fluid samples and scans.

\section{Clinical and cognitive data}

From all parent cohorts, clinical information and neuropsychological tests were collected centrally, harmonized, pooled and stored in an online data platform as previously described [13]. In short, all parent cohorts administered the Mini-Mental State Examination (MMSE), and performed neuropsychological testing covering various cognitive domains, although the tests used varied across the different cohorts. For the cognitive domains memory, language, attention, executive functioning and visuo-construction, one priority test was selected from each cohort (Additional file 1: Table S1) and $z$-scores were computed based on local normative data when available, or published normative data from healthy controls otherwise. 


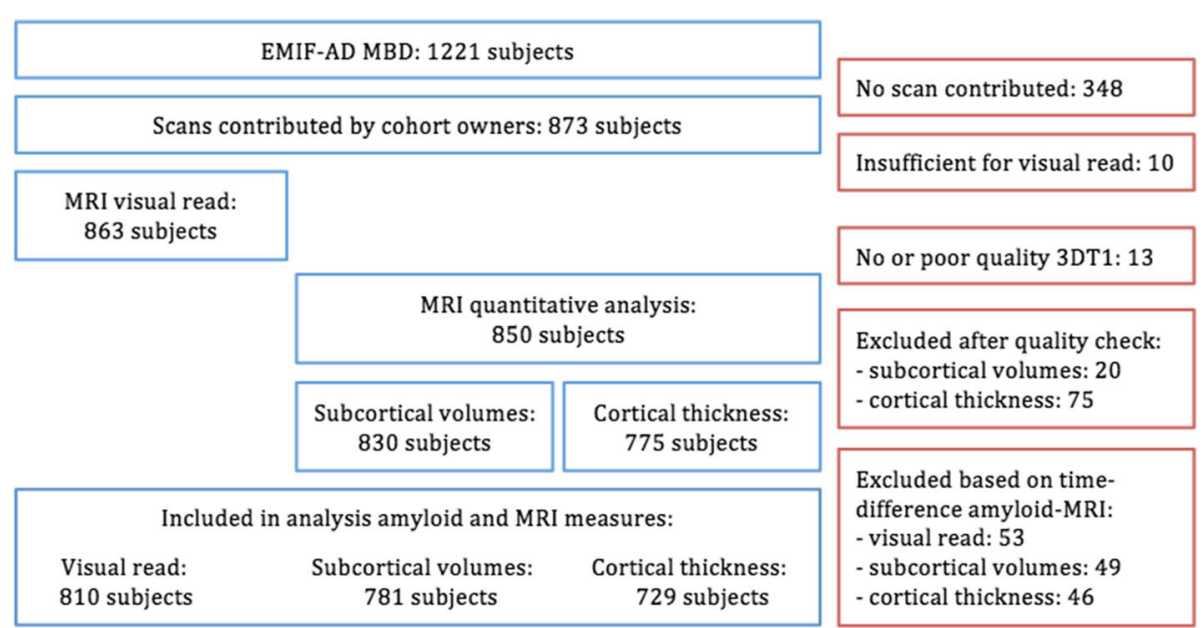

Fig. 1 Number of included subjects. EMIF-AD MBD European Medical Information Framework for Alzheimer's Disease Multimodal Biomarker Discovery, $M R I$ magnetic resonance imaging

\section{APOE genotyping}

For the entire EMIF-AD MBD cohort, $A P O E$ genotyping data from the local genetic analyses were available for 1121 (91\%) individuals. Central genetic analyses were performed at Lübeck University, Germany for 805 DNA and 148 whole blood samples. From the blood samples, DNA was extracted using the QIAamp ${ }^{\oplus}$ DNA Blood Mini Kit (QIAGEN GmbH, Hilden, Germany) resulting in 953 DNA samples, of which 926 passed quality control. Genome-wide SNP genotyping was performed using the Infinium Global Screening Array (GSA) with Shared Custom Content (Illumina Inc.). APOE genotypes were determined either directly (rs7212) or by imputation (rs429358). For 80 samples for which no local $A P O E$ genotype was available, and for 45 mismatches between local and GSA-derived genotypes, the APOE genotype was determined using TaqMan assays (ThermoFisher Scientific, Foster City, CA, USA) on a QuantStudio-12 K-Flex system. TaqMan re-genotyping confirmed 23 GSA genotypes and 21 local genotypes. For one failed sample we retained the local genotype. We classified individuals as $A P O E$ \&4 carriers or non-carriers according to their genotype status at rs429358 $(\mathrm{C}$-allele $=\varepsilon 4)$.

\section{Amyloid classification}

In the current selection $(n=863)$, amyloid status was defined by central analysis of CSF when available $(n=510)$, otherwise by local amyloid PET $(n=174)$ or local CSF $(n=179)$ measures. Central CSF analysis was performed at Gothenburg University, Sweden and included $A \beta_{1-40}$ and $A \beta_{1-42}$ measured using the V-PLEX Plus $A \beta$ Peptide Panel 1 (6E10) Kit (Meso Scale Discovery, Rockville, MD, USA), as described by the manufacturer. The central cut-off value for $A \beta$ positivity was an $A \beta_{42 / 40}$ ratio $<0.061$. Amyloid PET was performed in one cohort using $\left[{ }^{18} \mathrm{~F}\right] \mathrm{flu}$ temetamol according to local standardized procedures, with a standardized uptake value ratio (SUVR) cut-off value $>1.38$ used for abnormality [24]. In short, SUVR images were computed from spatially normalized summed images with cerebellar grey matter as the reference region. The cut-off value was derived from an independent dataset [25] and based on the statistical difference between $\mathrm{AD}$ dementia patients and cognitively normal subjects [24]. Local CSF amyloid was determined according to local protocols with local cut-off values. The number of amyloid positive subjects per diagnosis per cohort is presented in Additional file 1: Table S2.

\section{MRI acquisition}

At each site, imaging was acquired according to local protocols. From each parent cohort, we centrally collected the T1-weighted images, and if available also fluid-attenuated inversion recovery (FLAIR) and susceptibility weighted images (SWI) or T2\%, at the VU University Medical Center, where a visual quality check was performed. The acquired sequences and acquisition parameters for the T1-weighted scans for each cohort are presented in Additional file 1: Table S3. Usually, MRI was assessed at baseline together with baseline cognitive and amyloid measures. For 104 subjects there was more than a 1-year difference between MRI acquisition and amyloid assessment. In cases where amyloid was abnormal and acquired before MRI, this subject was included in the analysis $(n=42)$. In cases where amyloid was normal and acquired after MRI, this subject was included in the analysis $(n=9)$. All other cases were excluded $(n=53)$. For 99 subjects there was more than a 1-year difference between baseline cognitive assessment and MRI. For these cases, we did not use the cognitive data in the multi-variable analysis. Demographic differences between subjects who were included and excluded for differences in time between 
MRI and amyloid or cognitive assessment are presented in Additional file 1: Tables S4 and S5.

\section{MRI visual rating}

MRI scans with sufficient quality $(n=863)$ were visually rated by a single experienced rater, blinded to demographic information during rating. Medial temporal lobe atrophy (MTA) was assessed on coronal reconstructions of the T1-weighted images using a 5-point scale ranging from no atrophy (0) to end-stage atrophy (4) [26]. The MTA results from the left and right hemisphere were averaged. Global cortical atrophy (GCA) was assessed on transversal FLAIR or T1 images using a 4-point scale [27]. Posterior atrophy was assessed using a 4-point scale [28] and averaged over hemispheres. White matter hyperintensities were visually assessed on FLAIR images $(n=812)$ using the 4-point Fazekas scale (none, punctate, early confluent, confluent) [29]. Microbleeds were assessed on SWI and/or T2* images $(n=445)$ and defined as rounded hypointense homogeneous foci of up to $10 \mathrm{~mm}$ in diameter in the brain parenchyma. Microbleeds were dichotomized as present ( $\geq 1$ microbleeds) or absent (0 microbleeds).

\section{MRI quantitative analysis}

Good quality 3D T1 images $(n=850)$ were uploaded on the N4U platform (https://neugrid4you.eu/) for automated quantitative processing. Subcortical volumes, cortical thickness and surface area measures were estimated from 3D T1 MRI using Freesurfer (version 5.3.0, https://surfer.nmr.mgh.harvard.edu) as previously described [30]. All segmentations were visually inspected. We excluded data from 20 subjects for subcortical volumes (five due to complete failure of the algorithm and 15 due to segmentation errors) and from 75 subjects for cortical thickness and surface area (five due to complete failure of the algorithm, 66 due to segmentations errors of the cortical ribbon and four for other failures). Subcortical volumes were normalized by total intracranial volume (TIV). Cortical thickness and surface area were available for 68 regions according to the Desikan-Killiany atlas implemented in Freesurfer. Additionally, we computed two AD-signature meta-ROI measures that have previously been presented in the literature: one by Dickerson et al. [10] consisting of the average cortical thickness in angular, precuneus, supramarginal, superior frontal, superior parietal, temporal pole, inferior temporal, medial temporal and inferior frontal cortex; and one by Jack et al. [31] consisting of the surface-area weighted average mean cortical thickness in entorhinal, inferior temporal, middle temporal and fusiform regions.

\section{Statistical methods}

\section{Univariate analysis}

Univariate statistical analyses were performed in $\mathrm{R}$ (version 3.3.1). Comparisons of clinical characteristics between amyloid positive and negative subjects within each diagnostic group were performed using independent $t$ tests or Mann-Whitney $U$ tests for continuous variables and chi-square tests for categorical variables. Baseline comparisons in quantitative MRI measures between groups were performed with linear mixed models (continuous outcome measures) (lme4 package, version 1.1-12; lmerTest package 2.0-36), mixed effects ordered logistic regressions (ordinal outcome measures) (ordinal package, version 2015.6-28) and mixed effects logistic regressions (dichotomous outcome measures) (lme4 package). In each model, we entered amyloid status (negative, positive) and diagnosis (CN, MCI and $\mathrm{AD}$ ) and their interaction as fixed effects. Age (centred on mean), gender and $A P O E \varepsilon 4$ status were added as covariates. Cohort was added as a random intercept. The analyses were corrected within diagnostic group (in total 22 tests: five visual ratings, 14 subcortical volumes, three cortical thickness summary measures) for multiple hypothesis testing with the p.adjust() function using the false discovery rate, and indicated as $p_{\mathrm{FDR}}$.

\section{Multi-variable analysis}

To find the best multi-variable predictor of amyloid pathology, we used a supervised machine-learning approach based on SVM analysis. In SVM, two classes are separated by finding a hyperplane that maximizes the margin of separation between data points of each class in a high-dimensional feature space. SVMs are used extensively in neuroimaging as they have been shown to predict outcomes with high accuracy and possess the ability to model diverse and high-dimensional data [32]. We built a classifier to separate amyloid positive from amyloid negative subjects separately in the $\mathrm{CN}$ and $\mathrm{MCI}$ subgroups and, for the sake of completeness, also in the whole sample (including $\mathrm{CN}$, $\mathrm{MCI}$ and $\mathrm{AD}$-dementia patients). To address the imbalance between the number of amyloid positive and amyloid negative subjects in each diagnostic group, we adopted the re-weighting strategy [33]. That means we adjusted weights of each SVM feature inversely proportional to amyloid positive versus negative frequencies.

Machine-learning approach We used the python Scikitlearn library (version 0.19.1) to perform SVM classification [34]. To prevent overfitting (i.e. the classifier works perfectly on the training data, but is poorly generalizable to new data), we performed feature relevance evaluation and dimensionality reduction using a tree-based feature selection approach with a nested 10-fold cross-validation design [35, 36]. This was performed separately within each subgroup $(\mathrm{CN}, \mathrm{MCI}$ and whole sample).

The nested cross-validation consists of an inner loop for model building and parameter estimation, and an outer loop for model testing. Consequently, the dataset was divided into two parts: a training plus validation subset and 
a test subset. In the inner loop, SVM models were trained with varying SVM hyper-parameters (i.e. cost parameters $C$ and kernel function) based on a grid search, and a feature selection was performed using classification trees. The validation set was used to determine the SVM hyper-parameters over the grid of possible values. The performance of the resulting model, with optimized SVM hyper-parameters and features, was subsequently evaluated on the test set in the outer loop. For this outer loop, we used a 10-fold cross-validation scheme so that the data were divided into 10 equally sized parts. Nine of these were used as the training/validation set and one as the test set, and the 10 parts were permuted in each iteration of the outer loop so that each one was used for testing once. Finally, the SVM results were averaged over the 10 folds to estimate the predictive power of the proposed model on the whole dataset.

Feature selection As the input for the classifier, we used demographic information, neuropsychological information, $A P O E \& 4$ genotype and MRI measures (visual ratings, subcortical volumes, regional cortical thickness and regional surface area measures). To combine information measured on different scales, continuous demographic and MRI measures were normalized to $z$-scores. In the adopted treebased feature selection strategy, the Gini index was used to measure the relevance of each feature [37]. Features with a Gini index above the mean were kept, others were discarded. The complete list of features considered and selected, in the whole dataset and for $\mathrm{CN}$ and MCI separately, is reported in Additional file 1: Table S8.

Performance evaluation To assess the performance of the classifier, we computed the averaged receiver operating characteristic (ROC) area under the curve (AUC), specificity, sensitivity and accuracy for the testing datasets. We initially maximized the Youden index, and then also explored the results when setting the sensitivity at $80 \%, 85 \%$, $90 \%, 95 \%$ and $100 \%$. To assess the added value of combining different sources of information, we also built classifiers including only demographic information and a single other biomarker type (neuropsychological tests, APOE $\varepsilon 4$ genotype, MRI measures). Differences in AUC ROCs between classifiers were assessed with DeLong's test.

\section{Results}

\section{Demographic and cognitive comparisons}

We included 810 subjects divided over three diagnostic groups: $\mathrm{CN}(n=337)$, MCI $(n=375)$ and AD dementia $(n=98)$. Within the CN group, $92(27 \%)$ subjects were amyloid positive, in the MCI group 235 (63\%) and in the AD-dementia group 95 (97\%). Demographic and clinical data according to diagnosis and amyloid status are presented in Table 1. The amyloid positive MCI subjects were older and had lower cognitive scores compared to the amyloid negative $\mathrm{MCI}$ subjects. In $\mathrm{CN}$, there were no differences in age or cognition between amyloid positive and amyloid negative subjects. Amyloid positive subjects were more often $A P O E \varepsilon 4$ carriers in both the $\mathrm{MCI}$ and $\mathrm{CN}$ groups.

\section{Univariate association between MRI measures and amyloid pathology}

Within the MCI group, subjects with amyloid pathology had higher visual rating scores of medial temporal lobe atrophy, global cortical atrophy and parietal atrophy compared to amyloid negative subjects (Table 2). There were no differences in Fazekas score or presence of microbleeds. Amyloid positive MCI subjects had statistically significantly lower bilateral hippocampus, amygdala, thalamus, left caudate and right putamen volumes, and a trend towards lower right caudate $\left(p_{\text {uncorrected }}=0.08\right)$ and bilateral accumbens (both $p_{\text {uncorrected }}=0.07$ ) volumes compared to amyloid negative MCI subjects (Table 3). Amyloid positive MCI subjects also had lower whole brain average cortical thickness, as well as in the two AD-signature meta-ROIs, compared to amyloid negative MCI subjects.

In the $\mathrm{CN}$ group, amyloid positive subjects had statistically significantly lower right hippocampus, left amygdala, left thalamus and bilateral accumbens volumes compared to amyloid negative subjects. The effect of amyloid pathology on hippocampal volume was stronger in MCI subjects compared to $\mathrm{CN}$ subjects (significant interaction diagnosis $\times$ amyloid status). Amyloid positive $\mathrm{CN}$ subjects had lower values in the Jack AD-signature meta-ROI ( $\left.p_{\text {uncorrected }}=0.02\right)$, but not in the Dickerson AD-signature meta-ROI $\left(p_{\text {uncorrected }}=0.3\right)$ or whole brain average cortical thickness $\left(p_{\text {uncorrected }}=0.3\right) \mathrm{com}$ pared to amyloid negative $\mathrm{CN}$ subjects. There were no differences in visual rating scores between amyloid positive and amyloid negative $\mathrm{CN}$ subjects. All individual cortical thickness and surface area regions are presented in Additional file 1: Tables S6 and S7.

Compared to amyloid positive $\mathrm{CN}$ subjects, amyloid positive MCI subjects had lower bilateral hippocampal and amygdala volumes (all $p<0.001$ ) and lower whole brain average cortical thickness $(p=0.001)$, as well as in the two AD-signature meta-ROIs (both $p<0.001$ ).

\section{Multi-variable classifier results}

The features selected by the classifier in $\mathrm{CN}$ subjects, $\mathrm{MCI}$ subjects and the whole sample are presented in Additional file 1: Table S8. Across diagnoses, $A P O E$ \&4 genotype was the most important feature. Other relevant features selected across samples were age, the neuropsychological memory scores and various MRI measures such as hippocampus 
Table 1 Baseline characteristics by diagnosis and amyloid status

\begin{tabular}{|c|c|c|c|c|c|c|}
\hline \multirow[t]{2}{*}{ Characteristic } & \multicolumn{2}{|l|}{ Cognitively normal } & \multicolumn{2}{|c|}{ Mild cognitive impairment } & \multicolumn{2}{|c|}{ Alzheimer-type dementia } \\
\hline & Amyloid negative & Amyloid positive & Amyloid negative & Amyloid positive & Amyloid negative & Amyloid positive \\
\hline $\mathrm{N}, \%$ within diagnosis & $245(73)$ & $92(27)$ & $140(37)$ & $235(63)$ & $3(3)$ & $95(97)$ \\
\hline Age (years) & $66.1 \pm 7.2$ & $67.5 \pm 7.2$ & $67.3 \pm 8.0$ & $70.2 \pm 7.0^{* * *}$ & $63.1 \pm 8.0$ & $67.1 \pm 7.7$ \\
\hline Male gender & $120(49)$ & $47(51)$ & $73(52)$ & $105(45)$ & $3(100)$ & $48(51)$ \\
\hline Education (years) & $13.2 \pm 3.5$ & $12.8 \pm 3.8$ & $10.8 \pm 4.0$ & $11.1 \pm 3.7$ & $10.3 \pm 5.1$ & $11.1 \pm 3.3$ \\
\hline MMSE & $28.9 \pm 1.2$ & $28.8 \pm 1.2$ & $27.1 \pm 2.2$ & $26.0 \pm 2.6^{* * *}$ & $27.7 \pm 1.2$ & $22.4 \pm 4.0^{* *}$ \\
\hline Memory immediate & $0.10 \pm 1.00$ & $0.08 \pm 1.10$ & $-0.64 \pm 1.32$ & $-1.22 \pm 1.44^{* * *}$ & $-0.45 \pm 0.91$ & $-2.25 \pm 1.06$ \\
\hline Memory delayed & $0.25 \pm 1.01$ & $0.30 \pm 1.09$ & $-0.90 \pm 1.29$ & $-1.37 \pm 1.41^{* *}$ & $-0.96 \pm 1.33$ & $-2.28 \pm 1.04$ \\
\hline Language & $-0.21 \pm 1.01$ & $0.01 \pm 1.04$ & $-0.65 \pm 1.30$ & $-0.88 \pm 1.27$ & $-0.76 \pm 0.38$ & $-1.95 \pm 1.02^{*}$ \\
\hline Attention & $0.32 \pm 1.03$ & $0.26 \pm 0.89$ & $-0.74 \pm 1.79$ & $-0.81 \pm 1.63$ & $0.54 \pm 0.52$ & $-2.03 \pm 1.94^{*}$ \\
\hline Executive functioning & $0.35 \pm 1.09$ & $0.12 \pm 1.15$ & $-0.76 \pm 1.89$ & $-1.11 \pm 1.98^{*}$ & $0.46 \pm 0.32$ & $-2.49 \pm 2.46^{*}$ \\
\hline Visuo-construction & $-0.23 \pm 1.36$ & $-0.19 \pm 1.20$ & $0.18 \pm 1.46$ & $-0.30 \pm 1.66^{*}$ & $-0.59 \pm 2.09$ & $-1.30 \pm 2.00$ \\
\hline APOE ع4 genotype & $89(36)$ & $53(58)^{* * *}$ & $27(19)$ & $160(66)^{* * *}$ & $2(67)$ & $66(69)$ \\
\hline \multicolumn{7}{|l|}{ Available markers } \\
\hline Visual & $245(100)$ & $92(100)$ & $140(100)$ & $235(100)$ & $3(100)$ & $95(100)$ \\
\hline Subcortical volumes & $240(98)$ & $90(98)$ & $130(93)$ & $230(98)^{*}$ & $2(67)$ & $89(94)$ \\
\hline Cortical thickness & $232(95)$ & $88(96)$ & $119(85)$ & $200(85)$ & $2(67)$ & $88(93)$ \\
\hline
\end{tabular}

Data presented as mean \pm standard deviation or count (\%). Demographic characteristics based on maximum available data (visual rating) $A P O E$ apolipoprotein $E, M M S E$ Mini-Mental State Examination

${ }^{*} p<0.05,{ }^{* *} p<0.01,{ }^{* * *} p<0.001$, difference between amyloid positive and negative within diagnostic group

and amygdala volumes, as well as cortical thickness in temporal and parahippocampal regions (Fig. 2).

Combining the informative selected features in the SVM resulted in AUC $=0.81 \pm 0.06$ in $\mathrm{MCI}$ subjects, AUC $=0.74 \pm 0.08$ in $\mathrm{CN}$ subjects and $\mathrm{AUC}=0.85 \pm 0.05$ in the whole sample to classify amyloid positive versus amyloid negative subjects (Fig. 3; Additional file 2: Figure S1). In $\mathrm{MCI}$, the combined classifier including information from all modalities performed statistically significantly better than the classifiers based on demographic information combined with neuropsychology or imaging measures alone. The classifier including demographic variables and $A P O E \& 4$ genotype did not improve after additionally adding imaging and cognitive variables in $\mathrm{MCI}$. In $\mathrm{CN}$, the combined classifier including information from all modalities (demographics, cognitive, genetics and imaging) performed statistically significantly better than the classifiers including variables from only a subset of these modalities (Fig. 3; Additional file 2: Figure S2). The results from the SVM including only imaging variables are displayed in Additional file 2: Figure S3.

Table 4 presents the accuracy, sensitivity and specificity of the combined SVM in CN subjects, MCI subjects and the whole sample maximizing the Youden index, and at different levels of sensitivity. When optimizing both specificity and sensitivity, the positive predictive value for amyloid pathology was 0.84 in MCI (a $42 \%$ increase compared to the a priori probability (i.e. prevalence) of 0.59 ) and the negative predictive value was 0.62 . In $\mathrm{CN}$, the SVM obtained a positive predictive value of 0.41 (a $64 \%$ increase compared to the prevalence of 0.25 ) and a negative predictive value of 0.84 .

Table 2 Visual rating scores according to diagnosis and amyloid status

\begin{tabular}{|c|c|c|c|c|c|c|}
\hline \multirow[t]{2}{*}{ Score } & \multicolumn{2}{|l|}{ Cognitively normal } & \multicolumn{2}{|c|}{ Mild cognitive impairment } & \multicolumn{2}{|c|}{ Alzheimer-type dementia } \\
\hline & Amyloid negative & Amyloid positive & Amyloid negative & Amyloid positive & Amyloid negative & Amyloid positive \\
\hline$\overline{\text { MTA }}$ & $0(0-1)$ & $0(0-1)$ & $0.5(0-1)$ & $1(0.5-1.5)^{t \dagger}$ & $1(0.5-2)$ & $1(1-2)$ \\
\hline GCA & $0(0-1)$ & $0(0-1)$ & $0(0-1)$ & $1(0-1)^{\dagger+}$ & $1(0-2)$ & $1(1-1)$ \\
\hline Parietal & $1(0-1)$ & $0.5(0-1)$ & $1(0-1)$ & $1(0-1.63)^{\dagger}$ & $2(0-2)$ & $1(1-2)$ \\
\hline Fazekas & $1(0-1)$ & $1(0-1)$ & $1(0-1)$ & $1(1-2)$ & $1(1-1)$ & $1(0-2)$ \\
\hline Microbleeds present & $6(21 \%)$ & $4(20 \%)$ & $29(25 \%)$ & $56(29 \%)$ & $1(50 \%)$ & 17 (22\%) \\
\hline
\end{tabular}

Data presented as median (interquartile range) or count (\%)

$A P O E$ apolipoprotein E, FDR false discovery rate, GCA global cortical atrophy, MTA medial temporal lobe atrophy

${ }^{\dagger} p_{\mathrm{FDR}}<0.05,{ }^{+\dagger} p_{\mathrm{FDR}}<0.01$, difference between amyloid positive and negative within diagnostic group. Analyses corrected for age, gender, APOE $\varepsilon 4$ genotype and cohort 
Table 3 Quantitative MRI measures according to diagnosis and amyloid status

\begin{tabular}{|c|c|c|c|c|c|c|c|c|c|}
\hline \multirow[t]{2}{*}{ MRI measure } & \multicolumn{2}{|c|}{ Cognitively normal } & \multicolumn{2}{|c|}{ Mild cognitive impairment } & \multicolumn{2}{|c|}{ Alzheimer-type dementia } & \multicolumn{3}{|l|}{$F$ value } \\
\hline & $\begin{array}{l}\text { Amyloid } \\
\text { negative }\end{array}$ & $\begin{array}{l}\text { Amyloid } \\
\text { positive }\end{array}$ & $\begin{array}{l}\text { Amyloid } \\
\text { negative }\end{array}$ & $\begin{array}{l}\text { Amyloid } \\
\text { positive }\end{array}$ & $\begin{array}{l}\text { Amyloid } \\
\text { negative }\end{array}$ & $\begin{array}{l}\text { Amyloid } \\
\text { positive }\end{array}$ & Diagnosis & Amyloid & $\begin{array}{l}\text { Diagnosis } \times \\
\text { amyloid }\end{array}$ \\
\hline Hippocampus left & 3837 (39) & $3752(58)$ & $3638(46)$ & $3353(47)^{++\dagger}$ & $3051(340)$ & $3124(61)$ & $32.0^{* * *}$ & 0.7 & $3.5^{*}$ \\
\hline Hippocampus right & $3960(53)$ & $3830(66)^{\#}$ & $3760(57)$ & $3389(57)^{\dagger+\dagger}$ & 3905 (337) & $3172(70)^{\#}$ & $19.7^{* * *}$ & $12.3^{* * *}$ & $5.4^{* *}$ \\
\hline Amygdala left & $1501(36)$ & $1439(40)^{\#}$ & $1405(37)$ & $1294(37)^{\dagger+\dagger}$ & $1604(171)$ & $1188(42)^{\#}$ & $9.2^{* * *}$ & $11.0^{* *}$ & 2.5 \\
\hline Amygdala right & $1567(52)$ & $1535(55)$ & $1522(53)$ & $1398(53)^{\dagger+\dagger}$ & $1612(183)$ & $1290(57)$ & $4.6^{* *}$ & $6.7^{* *}$ & $3.2^{*}$ \\
\hline Thalamus left & $6834(101)$ & $6614(119)^{\#}$ & $6951(107)$ & $6689(108)^{\dagger}$ & $6187(564)$ & $6787(126)$ & 1.1 & 0.04 & 1.1 \\
\hline Thalamus right & $6388(104)$ & $6320(113)$ & $6419(106)$ & $6173(107)^{t+\dagger}$ & $5600(420)$ & $6185(117)$ & 2.4 & 0.4 & $3.2^{*}$ \\
\hline Caudate left & $3419(67)$ & $3336(80)$ & $3571(71)$ & $3407(71)^{\dagger}$ & 4151 (393) & $3387(85)$ & 2.9 & $6.1^{*}$ & 1.7 \\
\hline Caudate right & 3491 (88) & $3396(98)$ & $3584(91)$ & 3463 (91) & $4575(407)$ & $3413(103)^{\#}$ & $3.7^{*}$ & $10.7^{* *}$ & $3.4^{*}$ \\
\hline Putamen left & $4831(105)$ & 4779 (117) & 4689 (108) & 4609 (109) & $4692(478)$ & 4509 (122) & 2.1 & 0.4 & 0.1 \\
\hline Putamen right & $4627(121)$ & 4607 (130) & $4659(123)$ & $4461(124)^{\dagger}$ & $4524(444)$ & 4302 (134) & 0.6 & 0.9 & 1.5 \\
\hline Pallidum left & $1366(32)$ & $1412(37)$ & $1370(33)$ & $1385(33)$ & $1488(168)$ & $1390(39)$ & 0.3 & 0.05 & 0.6 \\
\hline Pallidum right & $1388(26)$ & $1382(31)$ & $1384(28)$ & $1379(28)$ & 1361 (148) & $1392(33)$ & 0.02 & 0.01 & 0.03 \\
\hline Accumbens left & $465(23)$ & $432(24)^{\#}$ & $434(23)$ & $411(23)$ & $526(79)$ & $375(25)$ & 2.1 & $6.7^{* *}$ & 1.5 \\
\hline Accumbens right & $497(22)$ & $467(23)^{\#}$ & $466(22)$ & $443(22)$ & $425(78)$ & 419 (24) & 2.3 & $4.0^{*}$ & 0.6 \\
\hline Average CT & $2.29(0.02)$ & $2.28(0.02)$ & $2.27(0.02)$ & $2.22(0.02)^{\dagger+t}$ & $2.22(0.07)$ & $2.19(0.02)$ & $5.3^{* *}$ & 1.6 & 1.5 \\
\hline CT Dickerson & $2.54(0.02)$ & $2.52(0.02)$ & $2.50(0.02)$ & $2.45(0.02)^{\dagger \dagger}$ & $2.48(0.09)$ & $2.38(0.02)$ & $8.3^{* * *}$ & 3.5 & 1.6 \\
\hline CT Jack & $2.68(0.03)$ & $2.63(0.03)^{\#}$ & $2.63(0.03)$ & $2.56(0.03)^{++\dagger}$ & $2.67(0.10)$ & $2.47(0.03)^{\#}$ & $6.7^{* *}$ & $9.3^{* *}$ & 1.7 \\
\hline
\end{tabular}

Data presented as estimate (standard error). Estimates derived from linear mixed models including diagnosis $\times$ amyloid, age, gender and $A P O E$ \&4 genotype as covariates and cohort as random effect

$A P O E$ apolipoprotein $\mathrm{E}, C T$ cortical thickness, FDR false discovery rate, MRI magnetic resonance imaging

${ }^{\dagger} p_{\mathrm{FDR}}<0.05,{ }^{\dagger+} p_{\mathrm{FDR}}<0.01,{ }^{++} p_{\mathrm{FDR}}<0.001,{ }^{\#} p_{\text {uncorrected }}<0.05$, compared to amyloid negative within diagnostic group

${ }^{*} p<0.05,{ }^{* *} p<0.01,{ }^{* * *} p<0.001$ for $F$ statistic of main effect

\section{Discussion}

In this study, we found that amyloid pathology is associated with brain atrophy in $\mathrm{CN}$ and MCI subjects. Using machine-learning techniques, we built a classifier based on a combination of demographic, cognitive, APOE $\varepsilon 4$ genotype and MRI data that could predict amyloid status at single subject level with a moderate level of accuracy. The performance of the classifier was higher in MCI subjects than in $\mathrm{CN}$ subjects. These results are of interest for clinical trial designers who wish to recruit amyloid positive subjects for inclusion.

Our results on the association between amyloid pathology and MRI measures in MCI are in line with previous studies that also found more cortical and subcortical atrophy in amyloid positive compared to amyloid negative MCI subjects [6, 7]. In $\mathrm{CN}$, amyloid pathology has previously been associated with cortical atrophy [9-11], and lower hippocampal volume in some studies $[8,9]$, but not in all $[11,38]$. To capture cortical changes associated with $\mathrm{AD}$, two different $\mathrm{AD}$-signature meta-ROIs have been proposed in the literature $[10,31]$. In MCI, both AD-signature measures were related to amyloid pathology. In $\mathrm{CN}$, only the AD-signature meta-ROI by Jack et al. [31] was associated with amyloid pathology in our study, suggesting that this one is more sensitive in the early disease stage. We also found an effect of amyloid pathology on nucleus accumbens volume, which was most pronounced in $\mathrm{CN}$ subjects. Although nucleus accumbens volumes are not often measured in AD-related studies, it has been hypothesized that this structure could show secondary neurodegeneration in $\mathrm{AD}$ in response to reduced input from connections to medial-temporal lobe structures [39]. It should be noted, however, that the nucleus accumbens is a small structure, which is difficult to segment automatically. These results require further validation in future studies.

The optimal features selected in the SVM by the tree-based approach included some, but not all, of the variables that showed differences between amyloid positive and amyloid negative subjects in the univariate analyses. Similarly, some of the features selected did not show statistically significant univariate group differences, although for many a trend towards lower values in amyloid positive subjects compared to amyloid negative subjects was observed. By combining the selected features derived from demographic information, neuropsychological examination, MRI measures and $A P O E \varepsilon 4$ genotype, we were able to classify $\mathrm{MCI}$ and $\mathrm{CN}$ subjects as amyloid positive or negative with a moderate level of accuracy.

The AUC for prediction of amyloid pathology was slightly higher in the MCI group compared to the $\mathrm{CN}$ 

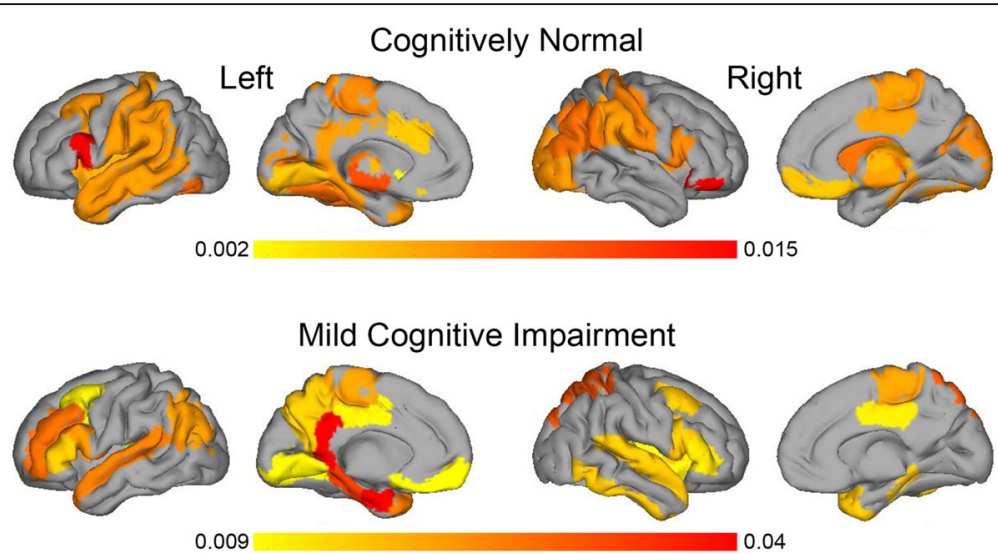

Fig. 2 Freesurfer regions selected as features for the classifier in cognitively normal (top row) and mild cognitive impairment (bottom row). Colour bars represent averaged feature weight

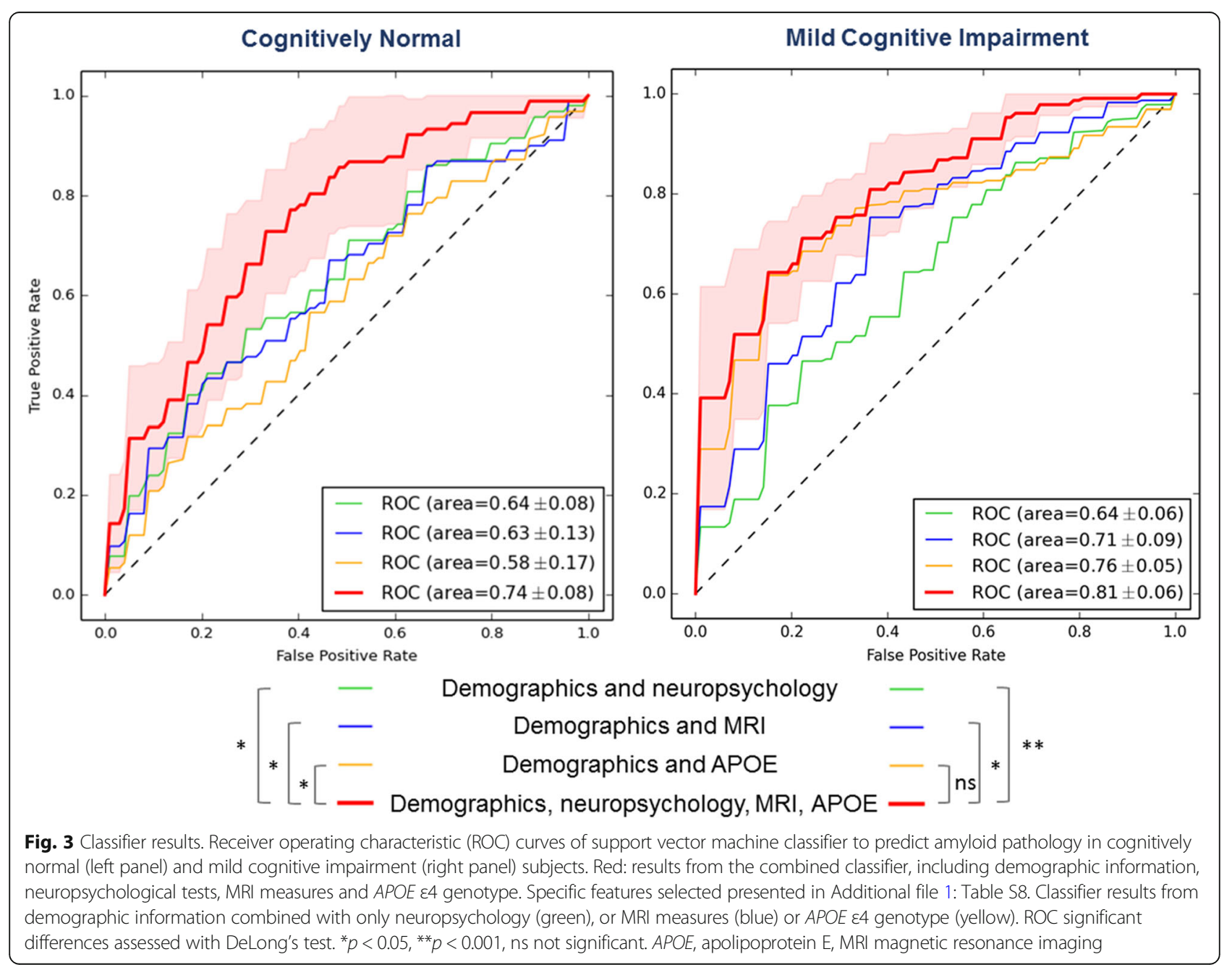


Table 4 Sensitivity, specificity, accuracy, PPV and NPV of the SVM classifier

\begin{tabular}{|c|c|c|c|c|c|c|}
\hline Group & Sensitivity & Specificity & Accuracy & PPV & NPV & Threshold SVM \\
\hline \multicolumn{7}{|c|}{ Optimized sensitivity and specificity } \\
\hline Cognitively normal & 0.61 & 0.71 & 0.68 & 0.41 & 0.84 & 0.70 \\
\hline Mild cognitive impairment & 0.71 & 0.77 & 0.74 & 0.84 & 0.62 & 0.33 \\
\hline Whole sample & 0.75 & 0.79 & 0.77 & 0.80 & 0.74 & 0.48 \\
\hline \multicolumn{7}{|l|}{$80 \%$ sensitivity } \\
\hline Cognitively normal & 0.80 & 0.55 & 0.62 & 0.41 & 0.88 & 0.77 \\
\hline Mild cognitive impairment & 0.80 & 0.64 & 0.74 & 0.79 & 0.66 & 0.47 \\
\hline Whole sample & 0.80 & 0.69 & 0.75 & 0.74 & 0.76 & 0.56 \\
\hline \multicolumn{7}{|l|}{$85 \%$ sensitivity } \\
\hline Cognitively normal & 0.85 & 0.46 & 0.57 & 0.38 & 0.89 & 0.80 \\
\hline Mild cognitive impairment & 0.85 & 0.54 & 0.73 & 0.75 & 0.68 & 0.53 \\
\hline Whole sample & 0.85 & 0.59 & 0.73 & 0.69 & 0.78 & 0.64 \\
\hline \multicolumn{7}{|l|}{$90 \%$ sensitivity } \\
\hline Cognitively normal & 0.90 & 0.36 & 0.51 & 0.35 & 0.91 & 0.84 \\
\hline Mild cognitive impairment & 0.90 & 0.46 & 0.73 & 0.73 & 0.74 & 0.60 \\
\hline Whole sample & 0.90 & 0.51 & 0.71 & 0.67 & 0.83 & 0.70 \\
\hline \multicolumn{7}{|l|}{ 95\% sensitivity } \\
\hline Cognitively normal & 0.95 & 0.24 & 0.44 & 0.32 & 0.92 & 0.87 \\
\hline Mild cognitive impairment & 0.95 & 0.37 & 0.73 & 0.71 & 0.83 & 0.70 \\
\hline Whole sample & 0.95 & 0.40 & 0.69 & 0.63 & 0.88 & 0.79 \\
\hline \multicolumn{7}{|l|}{$100 \%$ sensitivity } \\
\hline Cognitively normal & 1.00 & 0.11 & 0.36 & 0.30 & 1.00 & 0.91 \\
\hline Mild cognitive impairment & 1.00 & 0.08 & 0.66 & 0.64 & 1.00 & 0.87 \\
\hline Whole sample & 1.00 & 0.04 & 0.54 & 0.53 & 1.00 & 0.95 \\
\hline
\end{tabular}

Results from combined classifier, including demographic information, neuropsychological tests, MRI measures and APOE $\varepsilon 4$ genotype. Specific features selected presented in Additional file 1: Table S8. Values averaged across 10-fold cross-validation. Youden's $J$ statistic employed

$A P O E$ apolipoprotein E, MRI magnetic resonance imaging, NPV negative predictive value, $P P V$ positive predictive value, SVM support vector machine

group, and in line with a previous study in MCI [40]. In that study, a SVM classifier to predict amyloid pathology in subjects with $\mathrm{MCI}$ was also developed. Using cognitive data, hippocampal volume, $A P O E \varepsilon 4$ genotype and peripheral blood protein markers from the Alzheimer's Disease Neuroimaging Initiative (ADNI) dataset, they obtained AUC $=0.80$ for predicting amyloid pathology in subjects with MCI. In contrast to a previous study [12], we did not find that combining MRI markers with $A P O E \varepsilon 4$ genotype improved prediction of amyloid pathology in $\mathrm{MCI}$ over only including $A P O E \varepsilon 4$.

Our results in $\mathrm{CN}$ are comparable to the result from a similar study using data from the ADNI and a monocentric cohort [41]. In that study, a machine-learning-based classifier including demographic variables, $A P O E$ \&4 genotype, cognitive testing and structural MRI data reached an AUC of around 0.6 in $\mathrm{CN}$ subjects to predict amyloid positivity. Other studies have used combinations of demographic information, $A P O E \& 4$ genotype and cognitive testing (without imaging measures) to predict amyloid positivity in $\mathrm{CN}$
[42, 43]. They obtained positive predictive values of 0.65 and 0.63 for amyloid positivity, which was a $43-59 \%$ increase compared to the baseline prevalence in the cohort ( 0.41 and 0.44 respectively). In comparison, in our study we obtained a positive predictive value of 0.41 for amyloid pathology in $\mathrm{CN}$, with a baseline prevalence of 0.25 in our cohort, which is a $64 \%$ increase in predictive value. To recruit $1000 \mathrm{CN}$ subjects with amyloid pathology, using the classifier could reduce the number of subjects needing to undergo amyloid assessment from 3925 to 2439 , which is a $38 \%$ decrease. Assuming a cost of $€ 850$ for the pre-screening (including MRI, $A P O E$ genotyping and cognitive testing) and $€ 3500$ for an amyloid PET scan, using the classifier for pre-screening could reduce the total screening costs by nearly $€ 2$ million in this $\mathrm{CN}$ population. This example is based on an optimized sum of sensitivity and specificity (Youden index). For clinical trial design, it might be more interesting to optimize the sensitivity of the classifier, which would minimize the proportion of falsely excluded amyloid positive subjects, at the cost of the positive predictive value. 
As can be seen in Table 4, with increasing sensitivity (and higher negative predictive value), the positive predictive value of the classifier becomes lower, which would lead to increasing costs of pre-screening.

We chose SVM as a classification method for several reasons. First, it is based on a robust strategy (i.e. maximum-margin hyper-plane), which is considered to be one of the best to reduce the prediction error in a classification task [44, 45]. Second, only few parameters need to be tuned in order to make it fully operational, making SVM relatively easy to set up and use. Finally, it is particularly well suited for the separation of two classes (in this case, amyloid positive and amyloid negative).

A strength of our study is that, unlike previous studies [40-42], we performed our study in a heterogeneous cohort, in which data acquisition protocols were not standardized and different MR scanners and acquisition parameters were used. In this heterogeneous cohort, we showed a similar predictive accuracy compared to prospective research cohorts, which used standardized data acquisition protocols. This highlights the robustness of our approach and suggests that the results may also be generalizable to other cohorts. This will need to be tested in future studies. Our results may be of interest for studies recruiting subjects from parent cohorts to be included in (secondary) prevention studies targeting anti-amyloid therapeutics [4]. Our findings suggest that for individuals with $\mathrm{MCI}$, screening for amyloid positivity can best be done by age and $A P O E \varepsilon 4$ genotype, with limited added value of MRI. In CN, MRI measures have an added value above the other markers.

This study has some limitations. First, we used data acquired at various centres, which had different inclusion criteria for subjects and used different protocols for data collection. However, as already discussed, this also increased generalizability. Second, not everyone had the same measure of amyloid pathology. When possible, we used centralized analysis of the CSF $A \beta_{42 / 40}$ ratio to identify amyloid positivity, which has been shown to correlate highly with PET measures of amyloid pathology [46, 47]. For data from one cohort, we only had amyloid PET data available. Although CSF and PET measures are usually in good agreement, some studies have suggested that CSF values might become abnormal earlier than PET $[48,49]$. Finally, the same dataset was used to train and test the SVM classifier. Although nested $k$-fold cross-validation grants good generalizability of the SVM model [36], studies in independent datasets are needed to further validate our results.

\section{Conclusions}

Amyloid pathology is associated with structural MRI changes in $\mathrm{AD}$ typical regions in $\mathrm{CN}$ subjects and in subjects with MCI. We developed a classifier that can predict amyloid pathology at a single subject level using a combination of easily obtainable, non-invasive measures. Our results are of interest for trial designers who intend to recruit a large number of amyloid positive subjects. Implementing pre-screening procedures consisting of simple, non-invasive tests could substantially reduce screening failure rates. In future studies, the classifier might be improved by adding data from other minimally invasive tests, such as blood proteins and genetic markers [40]. In the EMIF-AD MBD study, plasma proteomics and metabolomics, and genomics and epigenomics, will also be analysed.

\section{Additional files}

Additional file 1: Additional Tables S1-S8 (PDF $264 \mathrm{~kb}$ )

Additional file 2: Additional Figures S1-S3 (PDF $770 \mathrm{~kb}$ )

\section{Abbreviations}

AD: Alzheimer's disease; APOE: Apolipoprotein E; CN: Cognitively normal; CSF: Cerebrospinal fluid; EMIF-AD MBD: European Medical Information Framework for Alzheimer's Disease Multimodal Biomarker Discovery; GCA: Global cortical atrophy visual rating; $\mathrm{MCl}$ : Mild cognitive impairment; MMSE: Mini-Mental State Examination; MRI: Magnetic resonance imaging; MTA: Medial temporal lobe atrophy visual rating; PET: Positron emission tomography; SVM: Support vector machine; TIV: Total intracranial volume

\section{Acknowledgements}

The authors acknowledge the contribution of the personnel of the Genomic Service Facility at the VIB-U Antwerp Center for Molecular Neurology.

\section{Funding}

The present study was conducted as part of the EMIF-AD project which has received support from the Innovative Medicines Initiative Joint Undertaking under EMIF grant agreement $n^{\circ} 115372$, resources of which are composed of a financial contribution from the European Union's Seventh Framework Programme (FP7/2007-2013) and an EFPIA companies' in-kind contribution. The DESCRIPA study was funded by the European Commission within the Fifth Framework Programme (QLRT-2001-2455). The EDAR study was funded by the European Commission within the Fifth Framework Programme (contract \# 37670). The VUmc Alzheimer Center is supported by Stichting Alzheimer Nederland and Stichting VUmc fonds, and the clinical database structure was developed with funding from Stichting Dioraphte. The Leuven cohort was funded by the Stichting voor Alzheimer Onderzoek (grant numbers \#11020, \#13007 and \#15005). The GAP study is supported by grants from the Department of Economic Promotion, Rural Areas and Territorial Balance of the Provincial Government of Gipuzkoa (124/16), the Department of Health of the Basque Government (2016111096), the Carlos III Institute of Health (PI15/00919, PN de I+ D + ( 2013-2016), Obra Social Kutxa-Fundazioa and anonymous private donors. The Gothenburg MCl study was supported by the Sahlgrenska University Hospital, Gothenburg, Sweden. The Lausanne cohort study was supported by a grant from the Swiss National Research Foundation to JP (SNF 320030_141179). The research at VIB-CMN is funded in part by the University of Antwerp Research Fund. RV is a senior clinical investigator of the Flemish Research Foundation (FWO). CVB is partly supported by the Flemish government-initiated Flanders Impulse Program on Networks for Dementia Research (VIND) and the Methusalem Excellence Program, the Research Foundation Flanders (FWO) and the University of Antwerp Research Fund, Belgium. FB is supported by the NIHR UCLH Biomedical Research Centre. $\mathrm{HZ}$ is supported by the Dementia Research Institute at $\mathrm{UCL}$ and is a Wallenberg Academy Fellow. SJV receives research support from ZonMw. WW has received funding from the European Union's Horizon 2020 Research and Innovation Programme under grant agreement no. 666992.

Availability of data and materials

The raw imaging data used in this study are stored on the online NeuGrid platform (https://neugrid4you.eu/). Clinical data used in this study and the derived imaging measures are stored on the online tranSMART platform 
from EMIF-AD. Access to the data is available upon request and after approval of each data provider, via the EMIF-AD platform.

\section{Authors' contributions}

MtK and AR provided data analyses, statistical analysis and data interpretation, and wrote the manuscript. EP provided data analyses. IB and SJV provided data management. RV, SG, JS, PS OB, JCR, RB, AW, CE, JLM, SE, CVB, PM-L, JP, MT and FRJV provided data and sample collection. ALB was responsible for plasma proteomics in EMIF-AD MBD. CL-Q was responsible for plasma metabolomics in EMIF-AD MBD. LB was responsible for genetic analyses in EMIF-AD MBD. VD was responsible for APOE analyses in EMIF-AD MBD. HZ was responsible for CSF analysis in EMIF-AD MBD. SL and JS provided study design and study coordination. SB provided data analyses. GPN is a member of the EMIF-AD MBD imaging workgroup and provided data interpretation. JR provided MRI data management. MFG and ZX are members of the EMIF-AD MBD imaging workgroup and provided data interpretation. WW provided critical revision of the manuscript. GF provided data and sample collection, and supervision of the project. PJV provided study design, study coordination and supervision of the project, and is a member of the EMIF-AD MBD imaging workgroup. FB provided study design, study coordination and supervision of the project, and is head of the EMIF-AD MBD imaging workgroup. All authors revised the manuscript and approved the final version.

\section{Ethics approval and consent to participate}

The local medical ethics committee in each centre approved the study. Subjects had already provided written informed consent at the time of inclusion in the cohort for use of data, samples and scans.

\section{Consent for publication}

Not applicable.

\section{Competing interests}

The authors declare that they have no competing interests. $\mathrm{HZ}$ has served on scientific advisory boards of Eli Lilly and Roche Diagnostics, has received travel support from Teva and is a co-founder of Brain Biomarker Solutions in Gothenburg AB, a GU Ventures-based platform company at the University of Gothenburg. MFG's current employer is Teva Pharmaceuticals, Inc., Malvern, PA, USA; his former employer was Boehringer Ingelheim Pharmaceuticals, Inc., Ridgefield, CT, USA. Any views expressed in this publication represent the personal opinions of the authors and not those of their respective employer. $J C R$ is a full-time employee of GlaxoSmithkline. PM-L reports personal fees from Lilly, Axon, General Electric and Nutricia for advisory boards, and lecturing fees from Lilly, Nutricia, Piramal. RV was principal investigator of the phase 1 and 2 $\left[{ }^{18} \mathrm{~F}\right]$ flutemetamol trials. RV's institution has clinical trial agreements (RV as PI) with AbbVie, Biogen, EliLilly, Merck and Novartis, and consultancy agreements (RV as PI) with Novartis and Cytox Ltd. SL has done consultancy for Eaisi, EIP Pharma, SomaLogic, Merck and Optum Labs.

\section{Publisher's Note}

Springer Nature remains neutral with regard to jurisdictional claims in published maps and institutional affiliations.

\footnotetext{
Author details

${ }^{1}$ Alzheimer Center \& Department of Neurology, VU University Medical Center, PO Box 7057, 1007 MB Amsterdam, the Netherlands. ${ }^{2}$ Laboratory of Epidemiology \& Neuroimaging, IRCCS San Giovanni di Dio Fatebenefratelli, Brescia, Italy. ${ }^{3}$ Alzheimer Centrum Limburg, Department of Psychiatry and Neuropsychology, Maastricht University, Maastricht, the Netherlands. ${ }^{4}$ University Hospital Leuven, Leuven, Belgium. ${ }^{5}$ Laboratory for Cognitive Neurology, Department of Neurosciences, KU Leuven, Leuven, Belgium. ${ }^{6} \mathrm{AP}-\mathrm{HM}, \mathrm{CHU}$ Timone, CIC CPCET, Service de Pharmacologie Clinique et Pharmacovigilance, Marseille, France. ${ }^{7}$ Neurosciences Therapeutic Area Unit, GlaxoSmithKline R\&D, Stevenage, UK. ${ }^{8}$ U1171 Inserm, CHU Lille, Degenerative and Vascular Cognitive Disorders, University of Lille, Lille, France. "Sahlgrenska Academy, Institute of Neuroscience and Physiology, Section for Psychiatry and Neurochemistry, University of Gothenburg, Gothenburg, Sweden. ${ }^{10}$ Barcelona ßeta Brain Research Center, Pasqual Maragall Foundation, Barcelona, Spain. ${ }^{11}$ Reference Center for Biological Markers of Dementia (BIODEM), Institute Born-Bunge, University of Antwerp, Antwerp, Belgium. ${ }^{12}$ Department of Neurology and Memory Clinic, Hospital Network Antwerp (ZNA) Middelheim and Hoge Beuken, Antwerp, Belgium.
}

${ }^{13}$ Neurodegenerative Brain Diseases, Center for Molecular Neurology, VIB, Antwerp, Belgium. ${ }^{14}$ Laboratory of Neurogenetics, Institute Born-Bunge, University of Antwerp, Antwerp, Belgium. ${ }^{15}$ Department of Neurology, Center for Research and Advanced Therapies, CITA-Alzheimer Foundation, San Sebastian, Spain. ${ }^{16}$ Department of Psychiatry, University Hospital of Lausanne, Lausanne, Switzerland. ${ }^{17}$ Geriatric Psychiatry, Department of Mental Health and Psychiatry, Geneva University Hospitals, Geneva, Switzerland. ${ }^{18}$ Memory and Dementia Center, 3rd Department of Neurology, "G Papanicolau" General Hospital, Aristotle University of Thessaloniki, Thessaloniki, Greece. ${ }^{19}$ University of Oxford, Oxford, UK. ${ }^{20}$ King's College London, London, UK. ${ }^{21}$ Lübeck Interdisciplinary Platform for Genome Analytics, University of Lübeck, Lubeck, Germany. ${ }^{22}$ School of Public Health, Imperial College London, London, UK. ${ }^{23}$ Department of Psychology, University of Oslo, Oslo, Norway. ${ }^{24}$ Department of Psychiatry and Neurochemistry, University of Gothenburg, Mölndal, Sweden. ${ }^{25}$ Department of Molecular Neuroscience, UCL Institute of Neurology, Queen Square, London, UK. ${ }^{26}$ UK Dementia Research Institute at UCL, London, UK. ${ }^{27}$ Clinical Neurochemistry Laboratory, Sahlgrenska University Hospital, Mölndal, Sweden. ${ }^{28}$ UCB Biopharma SPRL, Braine-l'Alleud, Belgium. ${ }^{29}$ Janssen Pharmaceutical Research and Development, Titusville, NJ, USA. ${ }^{30}$ MAAT, Archamps, France. ${ }^{31}$ Teva Pharmaceuticals, Inc., Malvern, PA, USA. ${ }^{32}$ Boehringer Ingelheim Pharmaceuticals, Inc., Ridgefield, CT, USA. ${ }^{33}$ Worldwide Research and Development, Pfizer Inc, Cambridge, MA, USA. ${ }^{34}$ Department of Radiology and Nuclear Medicine, VUMC, Amsterdam, the Netherlands. ${ }^{35}$ University of Geneva, Geneva, Switzerland. ${ }^{36}$ Institutes of Neurology and Healthcare Engineering, UCL, London, UK.

Received: 10 June 2018 Accepted: 4 September 2018 Published online: 27 September 2018

\section{References}

1. Scheltens P, Blennow K, Breteler MMB, de Strooper B, Frisoni GB, Salloway S, et al. Alzheimer's disease. Lancet. 2016;388:505-17.

2. Reiman EM, Langbaum JBS, Fleisher AS, Caselli RJ, Chen K, Ayutyanont N, et al. Alzheimer's Prevention Initiative: a plan to accelerate the evaluation of presymptomatic treatments. J Alzheimers Dis. 2011;26(Suppl 3):321-9.

3. Sperling RA, Rentz DM, Johnson KA, Karlawish J, Donohue M, Salmon DP, et al. The A4 study: stopping AD before symptoms begin? Sci Transl Med. 2014;6:228fs13.

4. Ritchie CW, Molinuevo JL, Truyen L, Satlin A, Van der Geyten S, Lovestone S, et al. Development of interventions for the secondary prevention of Alzheimer's dementia: the European Prevention of Alzheimer's Dementia (EPAD) project. Lancet Psychiatry. 2016;3:179-86.

5. Jansen WJ, Ossenkoppele R, Knol DL, Tijms BM, Scheltens P, Verhey FRJ, et al. Prevalence of cerebral amyloid pathology in persons without dementia: a meta-analysis. JAMA. 2015;313:1924-38.

6. Ten Kate M, Barkhof F, Visser PJ, Teunissen CE, Scheltens P, van der Flier WM, et al. Amyloid-independent atrophy patterns predict time to progression to dementia in mild cognitive impairment. Alzheimers Res Ther. 2017;9:73.

7. Huijbers W, Mormino EC, Schultz AP, Wigman S, Ward AM, Larvie M, et al. Amyloid- $\beta$ deposition in mild cognitive impairment is associated with increased hippocampal activity, atrophy and clinical progression. Brain. 2015;138:1023-35.

8. Hedden T, Dijk KRAV, Becker JA, Mehta A, Sperling RA, Johnson KA, et al. Disruption of functional connectivity in clinically normal older adults harboring amyloid burden. J Neurosci. 2009;29:12686-94.

9. Storandt M, Mintun M, Head D, Morris J. Cognitive decline and brain volume loss as signatures of cerebral amyloid- $\beta$ peptide deposition identified with Pittsburgh compound b: cognitive decline associated with A $\beta$ deposition. Arch Neurol. 2009;66:1476-81.

10. Dickerson BC, Bakkour A, Salat DH, Feczko E, Pacheco J, Greve DN, et al. The cortical signature of Alzheimer's disease: regionally specific cortical thinning relates to symptom severity in very mild to mild AD dementia and is detectable in asymptomatic amyloid-positive individuals. Cereb Cortex. 2009;19:497-510.

11. Becker JA, Hedden T, Carmasin J, Maye J, Rentz DM, Putcha D, et al. Amyloid- $\beta$ associated cortical thinning in clinically normal elderly. Ann Neurol. 2011;69:1032-42

12. Tosun D, Joshi S, Weiner MW. Alzheimer's Disease Neuroimaging Initiative. Neuroimaging predictors of brain amyloidosis in mild cognitive impairment. Ann Neurol. 2013;74:188-98. 
13. Bos I, Vos S, Vandenberghe R, Scheltens P, Engelborghs S, Frisoni G, et al. The EMIF-AD Multimodal Biomarker Discovery study: design, methods and cohort characteristics. Alzheimers Res Ther. 2018;10:64.

14. Visser PJ, Verhey FRJ, Boada M, Bullock R, Deyn PPD, Frisoni GB, et al. Development of screening guidelines and clinical criteria for predementia Alzheimer's disease. NED. 2008:30:254-65.

15. Reijs BLR, Ramakers IHGB, Köhler S, Teunissen CE, Koel-Simmelink M, Nathan PJ, et al. Memory correlates of Alzheimer's disease cerebrospinal fluid markers: a longitudinal cohort study. J Alzheimers Dis. 2017;60:1119-28.

16. Galluzzi S, Marizzoni M, Babiloni C, Albani D, Antelmi L, Bagnoli C, et al. Clinical and biomarker profiling of prodromal Alzheimer's disease in workpackage 5 of the Innovative Medicines Initiative PharmaCog project: a 'European ADNI study'. J Intern Med. 2016;279:576-91.

17. van der Flier WM, Pijnenburg YAL, Prins N, Lemstra AW, Bouwman FH, Teunissen $C E$, et al. Optimizing patient care and research: the Amsterdam Dementia Cohort. J Alzheimers Dis. 2014;41:313-27.

18. Ten Kate M, Sanz-Arigita EJ, Tijms BM, Wink AM, Clerigue M, GarciaSebastian M, et al. Impact of APOE- $\varepsilon 4$ and family history of dementia on gray matter atrophy in cognitively healthy middle-aged adults. Neurobiol Aging. 2016;38:14-20.

19. Somers C, Struyfs H, Goossens J, Niemantsverdriet E, Luyckx J, De Roeck N, et al. A decade of cerebrospinal fluid biomarkers for Alzheimer's disease in Belgium. J Alzheimers Dis. 2016;54:383-95.

20. Adamczuk K, De Weer A-S, Nelissen N, Dupont P, Sunaert S, Bettens K, et al. Functional changes in the language network in response to increased amyloid $\beta$ deposition in cognitively intact older adults. Cereb Cortex. 2017; 27:3879.

21. Oikonomidi A, Tautvydaite D, Gholamrezaee MM, Henry H, Bacher M, Popp J. Macrophage migration inhibitory factor is associated with biomarkers of Alzheimer's disease pathology and predicts cognitive decline in mild cognitive impairment and mild dementia. J Alzheimers Dis. 2017;60:273-81.

22. Wallin A, Nordlund A, Jonsson M, Lind K, Edman Å, Göthlin M, et al. The Gothenburg MCl study: design and distribution of Alzheimer's disease and subcortical vascular disease diagnoses from baseline to 6-year follow-up. J Cereb Blood Flow Metab. 2016;36:114-31.

23. Fortea J, Sala-Llonch R, Bartrés-Faz D, Bosch B, Lladó A, Bargalló N, et al. Increased cortical thickness and caudate volume precede atrophy in PSEN1 mutation carriers. J Alzheimers Dis. 2010;22:909-22.

24. Adamczuk K, Schaeverbeke J, Nelissen N, Neyens V, Vandenbulcke M, Goffin $\mathrm{K}$, et al. Amyloid imaging in cognitively normal older adults: comparison between (18)F-flutemetamol and (11)C-Pittsburgh compound B. Eur J Nucl Med Mol Imaging. 2016:43:142-51.

25. Vandenberghe R, Van Laere K, Ivanoiu A, Salmon E, Bastin C, Triau E, et al. 18F-flutemetamol amyloid imaging in Alzheimer disease and mild cognitive impairment: a phase 2 trial. Ann Neurol. 2010;68:319-29.

26. Scheltens $P$, Leys $D$, Barkhof $F$, Huglo D, Weinstein HC, Vermersch $P$, et al. Atrophy of medial temporal lobes on MRI in "probable" Alzheimer's disease and normal ageing: diagnostic value and neuropsychological correlates. J Neurol Neurosurg Psychiatry. 1992;55:967-72.

27. Pasquier F, Leys D, Weerts JG, Mounier-Vehier F, Barkhof F, Scheltens P. Inter- and intraobserver reproducibility of cerebral atrophy assessment on MRI scans with hemispheric infarcts. Eur Neurol. 1996;36:268-72.

28. Koedam ELGE, Lehmann $M$, van der Flier WM, Scheltens P, YAL P, Fox N, et al. Visual assessment of posterior atrophy development of a MRI rating scale. Eur Radiol. 2011;21:2618-25.

29. Fazekas F, Chawluk JB, Alavi A, Hurtig HI, Zimmerman RA. MR signal abnormalities at 1.5 T in Alzheimer's dementia and normal aging. Am J Neuroradiol. 1987:8:421-6.

30. Fischl B. FreeSurfer. Neuroimage. 2012;62:774-81.

31. Jack CR, Wiste HJ, Weigand SD, Therneau TM, Lowe VJ, Knopman DS, et al. Defining imaging biomarker cut points for brain aging and Alzheimer's disease. Alzheimers Dement. 2017;13:205-16

32. Orrù G, Petterson-Yeo W, Marquand AF, Sartori G, Mechelli A. Using support vector machine to identify imaging biomarkers of neurological and psychiatric disease: a critical review. Neurosci Biobehav Rev. 2012;36:1140-52.

33. Chang C-C, Lin C-J. LIBSVM: A Library for Support Vector Machines. ACM Trans Intell Syst Technol. 2011;2:27 1-27:27.

34. Pedregosa F, Varoquaux G, Gramfort A, Michel V, Thirion B, Grisel O, et al. Scikitlearn: Machine Learning in Python. J Mach Learn Res. 2011;12:2825-30.

35. Ambroise C, McLachlan GJ. Selection bias in gene extraction on the basis of microarray gene-expression data. Proc Natl Acad Sci U S A. 2002;99:6562-6.
36. Cawley GC, Talbot NLC. On over-fitting in model selection and subsequent selection bias in performance evaluation. J Mach Learn Res. 2010;11:2079-107.

37. Cutler A, Cutler DR, Stevens JR. Random Forests. In: Zhang C, Ma Y, editors. Ensemble Machine Learning: Methods and Applications [Internet]. Boston: Springer US; 2012. p. 157-75. Available from: https://doi.org/10.1007/978-14419-9326-7_5.

38. Mattsson N, Insel PS, Nosheny R, Tosun D, Trojanowski JQ, Shaw LM, et al. Emerging $\beta$-amyloid pathology and accelerated cortical atrophy. JAMA Neurol. 2014;71:725-34.

39. Pievani M, Bocchetta M, Boccardi M, Cavedo E, Bonetti M, Thompson PM, et al. Striatal morphology in early-onset and late-onset Alzheimer's disease: a preliminary study. Neurobiol Aging. 2013;34:1728-39.

40. Apostolova LG, Hwang KS, Avila D, Elashoff D, Kohannim O, Teng E, et al. Brain amyloidosis ascertainment from cognitive, imaging, and peripheral blood protein measures. Neurology. 2015:84:729-37.

41. Ansart M, Epelbaum S, Gagliardi G, Colliot O, Dormont D, Dubois B, et al. Prediction of Amyloidosis from Neuropsychological and MRI Data for Cost Effective Inclusion of Pre-symptomatic Subjects in Clinical Trials. Deep Learning in Medical Image Analysis and Multimodal Learning for Clinical Decision Support [Internet]. Cham: Springer; 2017. p. 357-64. [cited 2018 Jan 11] Available from: https://link.springer.com/chapter/10.1007/978-3-31967558-9_41

42. Insel PS, Palmqvist S, Mackin RS, Nosheny RL, Hansson O, Weiner MW, et al. Assessing risk for preclinical $\beta$-amyloid pathology with $\mathrm{APOE}$, cognitive, and demographic information. Alzheimers Dement. 2016;4:76-84.

43. Mielke MM, Wiste HJ, Weigand SD, Knopman DS, Lowe VJ, Roberts RO, et al. Indicators of amyloid burden in a population-based study of cognitively normal elderly. Neurology. 2012;79:1570-7.

44. Burges CJC. A tutorial on support vector machines for pattern recognition. Data Min Knowl Disc. 1998;2:121-67.

45. Kounelakis M, Zervakis M, Kotsiakis X. Chapter 13-the impact of microarray technology in brain cancer. In: AFG T, Fisher AC, editors. Outcome Prediction in Cancer [Internet]. Amsterdam: Elsevier; 2007. p. 339-88. [cited 2018 Aug 1] Available from: http://www.sciencedirect.com/science/article/ pii/B9780444528551500155.

46. Janelidze S, Pannee J, Mikulskis A, Chiao P, Zetterberg H, Blennow K, et al. Concordance between different amyloid immunoassays and visual amyloid positron emission tomographic assessment. JAMA Neurol. 2017;74:1492-501.

47. Lewczuk P, Matzen A, Blennow K, Parnetti L, Molinuevo JL, Eusebi P, et al. Cerebrospinal fluid $A \beta 42 / 40$ corresponds better than $A \beta 42$ to amyloid PET in Alzheimer's disease. J Alzheimers Dis. 2017;55:813-22.

48. Mattsson N, Insel PS, Donohue M, Landau S, Jagust WJ, Shaw LM, et al. Independent information from cerebrospinal fluid amyloid- $\beta$ and florbetapir imaging in Alzheimer's disease. Brain. 2015;138:772-83.

49. Palmqvist $\mathrm{S}$, Mattsson N, Hansson O. Alzheimer's Disease Neuroimaging Initiative. Cerebrospinal fluid analysis detects cerebral amyloid- $\beta$ accumulation earlier than positron emission tomography. Brain. 2016;139: $1226-36$.
Ready to submit your research? Choose BMC and benefit from:
- fast, convenient online submission
- thorough peer review by experienced researchers in your field
- rapid publication on acceptance
- support for research data, including large and complex data types
- gold Open Access which fosters wider collaboration and increased citations
- maximum visibility for your research: over $100 \mathrm{M}$ website views per year
At BMC, research is always in progress.
Learn more biomedcentral.com/submissions 